Mathematical Modelling and Analysis

Volume 19 Number 1, February 2014, 52-65

http://dx.doi.org/10.3846/13926292.2014.893261

(c) Vilnius Gediminas Technical University, 2014
Publisher: Taylor\&Francis and VGTU

http://www.tandfonline.com/TMMA

Print ISSN: 1392-6292

Online ISSN: 1648-3510

\title{
A Mixed Joint Universality Theorem for Zeta-Functions. II
}

\author{
Vaida Pocevičienè ${ }^{a}$ and Darius Šiaučiūnas ${ }^{b}$ \\ ${ }^{a}$ Faculty of Technologies, Panevezzys Institute, Kaunas University of \\ Technology \\ S. Daukanto str. 12, LT-35212 Panevėžys, Lithuania \\ ${ }^{b}$ Faculty of Mathematics and Informatics, Šiauliai University \\ P. Višinskio str. 19, LT-77156 Šiauliai, Lithuania \\ E-mail(corresp.): siauciunas@fm.su.lt \\ E-mail: vaida.poceviciene@ktu.lt
}

Received June 28, 2013; revised December 26, 2013; published online February 20, 2014

\begin{abstract}
In the paper, a joint universality theorem on the approximation of analytic functions for zeta-function of a normalized Hecke eigen cusp form and a collection of periodic Hurwitz zeta-functions with algebraically independent parameters is obtained.
\end{abstract}

Keywords: Hurwitz zeta-function, universality, zeta-function of certain cusp form.

AMS Subject Classification: $11 \mathrm{M} 41$.

Let $\mathfrak{a}=\left\{a_{m}: m \in \mathbb{N}_{0}=\mathbb{N} \cup\{0\}\right\}$ be a periodic sequence of complex numbers with minimal period $k \in \mathbb{N}, \alpha, 0<\alpha \leq 1$, be a fixed parameter, and $s=\sigma+i t$. The periodic Hurwitz zeta-function $\zeta(s, \alpha ; \mathfrak{a})$ is defined, for $\sigma>1$, by

$$
\zeta(s, \alpha ; \mathfrak{a})=\sum_{m=0}^{\infty} \frac{a_{m}}{(m+\alpha)^{s}},
$$

and is analytically continued to an entire function if

$$
a \stackrel{\text { def }}{=} \frac{1}{k} \sum_{l=0}^{k-1} a_{l}=0,
$$

while if $a \neq 0$, then $\zeta(s, \alpha ; \mathfrak{a})$ is a meromorphic function having the unique simple pole at $s=1$ with residue $a$.

In [4], a joint universality theorem for the Riemann zeta-function $\zeta(s)$ and a collection of periodic Hurwitz zeta-functions has been obtained. For $j=$ $1, \ldots, r$ let $\alpha_{j}, 0<\alpha_{j} \leq 1$, be a fixed parameter, $l_{j} \in \mathbb{N}$, and, for $j=1, \ldots, r$, $l=1, \ldots, l_{j}$, let $\mathfrak{a}_{j l}=\left\{a_{m j l}: m \in \mathbb{N}_{0}\right\}$ be a periodic sequence of complex 
numbers with minimal period $k_{j l}$, and $\zeta\left(s, \alpha_{j} ; \mathfrak{a}_{j l}\right)$ denote the corresponding periodic Hurwitz zeta-function. Denote by $k_{j}$ the least common multiple of the periods $k_{j 1}, \ldots, k_{j l_{j}}$, and define

$$
B_{j}=\left(\begin{array}{cccc}
a_{1 j 1} & a_{1 j 2} & \ldots & a_{1 j l_{j}} \\
a_{2 j 1} & a_{2 j 2} & \ldots & a_{2 j l_{j}} \\
\ldots & \ldots & \ldots & \ldots \\
a_{k_{j} j 1} & a_{k_{j} j 2} & \ldots & a_{k_{j} j l_{j}}
\end{array}\right), \quad j=1, \ldots, r .
$$

Let $D=\left\{s \in \mathbb{C}: \frac{1}{2}<\sigma<1\right\}$, and let, for brevity,

$$
\nu_{T}(\ldots)=\frac{1}{T} \operatorname{meas}\{\tau \in[0, T]: \ldots\},
$$

where meas $A$ denotes the Lebesgue measure of a measurable set $A \subset \mathbb{R}$, and in the place of dots a condition satisfied by $\tau$ is to be written. Then the main result of [4] is contained in the following theorem.

Theorem 1. Suppose that the numbers $\alpha_{1}, \ldots, \alpha_{r}$ are algebraically independent over the field of rational numbers $\mathbb{Q}$, and that $\operatorname{rank}\left(B_{j}\right)=l_{j}, j=1, \ldots, r$. For every $j=1, \ldots, r$ and $l=1, \ldots, l_{j}$, let $K_{j l}$ be a compact subset of the strip $D$ with connected complement, and let $f_{j l}(s)$ be a continuous on $K_{j l}$ function which is analytic in the interior of $K_{j l}$. Moreover, let $K$ be a compact subset of the strip $D$ with connected complement, and $f(s)$ be a continuous non-vanishing on $K$ function which is analytic in the interior of $K$. Then, for every $\varepsilon>0$,

$$
\begin{aligned}
& \liminf _{T \rightarrow \infty} \nu_{T}\left(\sup _{s \in K}|\zeta(s+i \tau)-f(s)|<\varepsilon,\right. \\
& \left.\quad \sup _{1 \leq j \leq r} \sup _{1 \leq j \leq l_{j}} \sup _{s \in K_{j l}}\left|\zeta\left(s+i \tau, \alpha_{j} ; \mathfrak{a}_{j l}\right)-f_{j l}(s)\right|<\varepsilon\right)>0 .
\end{aligned}
$$

A natural question arises if the Riemann zeta-function in Theorem 1 can be replaced by other zeta-functions which are universal in a certain strip?

Let $F$ be a normalized Hecke eigen cusp form of weight $\kappa$ for the full modular group, and let

$$
F(z)=\sum_{m=1}^{\infty} c(m) \mathrm{e}^{2 \pi i m z}
$$

be its Fourier series expansion. The zeta-function $\varphi(s, F)$ attached to the form $F$ is defined, for $\sigma>\frac{\kappa+1}{2}$, by

$$
\varphi(s, F)=\sum_{m=1}^{\infty} \frac{c(m)}{m^{s}},
$$

and is analytically continued to an entire function. Moreover, for $\sigma>\frac{\kappa+1}{2}$, the function $\varphi(s, F)$ has the Euler product over primes

$$
\varphi(s, F)=\prod_{p}\left(1-\frac{\alpha(p)}{p^{s}}\right)^{-1}\left(1-\frac{\beta(p)}{p^{s}}\right)^{-1},
$$


where $\alpha(p)$ and $\beta(p)$ are complex conjugate numbers such that $\alpha(p)+\beta(p)=$ $c(p)$, and

$$
|\alpha(p)|<p^{\frac{\kappa-1}{2}}, \quad|\beta(p)| \leq p^{\frac{\kappa-1}{2}} .
$$

In [5], the universality of the function $\varphi(s, F)$ has been obtained. Let $D_{\kappa}=$ $\left\{s \in \mathbb{C}: \frac{\kappa}{2}<\sigma<\frac{\kappa+1}{2}\right\}$.

Theorem 2. [5] Let $K$ be a compact subset of the strip $D_{\kappa}$ with connected complement, and let $f(s)$ be a continuous non-vanishing function on $K$, and analytic in the interior of $K$. Then, for every $\varepsilon>0$,

$$
\liminf _{T \rightarrow \infty} \nu_{T}\left(\sup _{s \in K}|\varphi(s+i \tau, F)-f(s)|<\varepsilon\right)>0 .
$$

The main result of the present paper connects Theorems 1 and 2 .

Theorem 3. Suppose that the numbers $\alpha_{1}, \ldots, \alpha_{r}$ are algebraically independent over $\mathbb{Q}$, and that $\operatorname{rank}\left(B_{j}\right)=l_{j}, j=1, \ldots, r$. Let $K_{j l}$ and $f_{j l}$ be the same as in Theorem 1, and $K$ and $f(s)$ be the same as in Theorem 2. Then, for every $\varepsilon>0$,

$$
\begin{aligned}
& \quad \liminf _{T \rightarrow \infty} \nu_{T}\left(\sup _{s \in K}|\varphi(s+i \tau, F)-f(s)|<\varepsilon,\right. \\
& \left.\quad \sup _{1 \leq j \leq r} \sup _{1 \leq l \leq l_{j}} \sup _{s \in K_{j l}}\left|\zeta\left(s+i \tau, \alpha_{j} ; \mathfrak{a}_{j l}\right)-f_{j l}(s)\right|<\varepsilon\right)>0 .
\end{aligned}
$$

For the proof of Theorem 3, we apply the probabilistic approach based on a joint limit theorem in the space of analytic functions. Theorem 3 is the first result on the joint universality for zeta-functions which presents the universality property in two different strips $D$ and $D_{\kappa}$. This is the novelty of the paper.

\section{Functional Limit Theorems}

For a region $G$ on the complex plane, let us denote by $H(G)$ the space of analytic functions on $G$ equipped with the topology of uniform convergence on compacta. Let

$$
H^{v}\left(D_{\kappa}, D\right)=H\left(D_{\kappa}\right) \times \underbrace{H(D) \times \cdots \times H(D)}_{v_{1}}, \quad v_{1}=\sum_{j=1}^{r} l_{j}, \quad v=v_{1}+1 .
$$

For brevity, we set

$$
\underline{\alpha}=\left(\alpha_{1}, \ldots, \alpha_{r}\right), \quad \underline{\mathfrak{a}}=\left(\mathfrak{a}_{11}, \ldots, \mathfrak{a}_{1 l_{1}}, \ldots, \mathfrak{a}_{r 1}, \ldots, \mathfrak{a}_{r l_{r}}\right)
$$

and

$$
\begin{aligned}
\underline{\zeta}(\hat{s}, s, \underline{\alpha} ; \underline{\mathfrak{a}}, F)= & \left(\varphi(\hat{s}, F), \zeta\left(s, \alpha_{1} ; \mathfrak{a}_{11}\right), \ldots, \zeta\left(s, \alpha_{1} ; \mathfrak{a}_{1 l_{1}}\right), \ldots,\right. \\
& \left.\zeta\left(s, \alpha_{r} ; \mathfrak{a}_{r 1}\right), \ldots, \zeta\left(s, \alpha_{r} ; \mathfrak{a}_{r l_{r}}\right)\right) .
\end{aligned}
$$


Denote by $\mathcal{B}(S)$ the class of Borel sets of the space $S$. In this section, we consider the weak convergence of the probability measure

$$
P_{T}(A) \stackrel{\text { def }}{=} \nu_{T}(\underline{\zeta}(\hat{s}+i \tau, s+i \tau, \underline{\alpha} ; \underline{\mathfrak{a}}, F) \in A), \quad A \in \mathcal{B}\left(H^{v}\left(D_{\kappa}, D\right)\right),
$$

as $T \rightarrow \infty$. To state a limit theorem, we need some notation.

Denote by $\gamma$ the unit circle on the complex plane, and define

$$
\hat{\Omega}=\prod_{p} \gamma_{p} \quad \text { and } \quad \Omega=\prod_{m=0}^{\infty} \gamma_{m}
$$

where $\gamma_{p}=\gamma$ for all primes $p$, and $\gamma_{m}=\gamma$ for all $m \in \mathbb{N}_{0}$. By the Tikhonov theorem, the tori $\hat{\Omega}$ and $\Omega$ are compact topological Abelian groups. Therefore, on $(\hat{\Omega}, \mathcal{B}(\hat{\Omega}))$ and $(\Omega, \mathcal{B}(\Omega))$ the probability Haar measures $\hat{m}_{H}$ and $m_{H}$, respectively, can be defined. We obtain two probability spaces $\left(\hat{\Omega}, \mathcal{B}(\hat{\Omega}), \hat{m}_{H}\right)$ and $\left(\Omega, \mathcal{B}(\Omega), m_{H}\right)$.

Furthermore, we put $\underline{\Omega}=\hat{\Omega} \times \Omega_{1} \times \cdots \times \Omega_{r}$, where $\Omega_{j}=\Omega$ for $j=1, \ldots, r$. Then the Tikhonov theorem implies again that $\underline{\Omega}$ is a compact topological Abelian group, and, similarly as above, we obtain one more probability space $\left(\underline{\Omega}, \mathcal{B}(\underline{\Omega}), \underline{m}_{H}\right)$, where $\underline{m}_{H}$ is the probability Haar measure on $(\underline{\Omega}, \mathcal{B}(\underline{\Omega}))$. Denote by $\hat{\omega}(p)$ the projection of $\hat{\omega} \in \Omega$ to the coordinate space $\gamma_{p}, p \in \mathcal{P},(\mathcal{P}$ is the set of all prime numbers), and by $\omega_{j}(m)$ the projection of $\omega_{j} \in \Omega_{j}$ to the coordinate space $\gamma_{m}, m \in \mathbb{N}_{0}$. Let $\underline{\omega}=\left(\hat{\omega}, \omega_{1}, \ldots, \omega_{r}\right)$ stand for elements of $\underline{\Omega}$. On the probability space $\left(\underline{\Omega}, \mathcal{B}(\underline{\Omega}), \underline{m}_{H}\right)$, define the $H^{v}\left(D_{\kappa}, D\right)$-valued random element $\underline{\zeta}(\hat{s}, s, \underline{\alpha}, \underline{\omega} ; \underline{\mathfrak{a}}, F)$ by the formula

$$
\begin{aligned}
\underline{\zeta}(\hat{s}, s, \underline{\alpha}, \underline{\omega} ; \underline{\mathfrak{a}}, F)= & \left(\varphi(\hat{s}, \hat{\omega}, F), \zeta\left(s, \alpha_{1}, \omega_{1} ; \mathfrak{a}_{11}\right), \ldots,\right. \\
& \left.\zeta\left(s, \alpha_{1}, \omega_{1} ; \mathfrak{a}_{1 l_{1}}\right), \ldots, \zeta\left(s, \alpha_{r}, \omega_{r} ; \mathfrak{a}_{r 1}\right), \ldots, \zeta\left(s, \alpha_{r}, \omega_{r} ; \mathfrak{a}_{r l_{r}}\right)\right),
\end{aligned}
$$

where

$$
\varphi(\hat{s}, \hat{\omega}, F)=\prod_{p}\left(1-\frac{\alpha(p) \hat{\omega}(p)}{p^{s}}\right)^{-1}\left(1-\frac{\beta(p) \hat{\omega}(p)}{p^{s}}\right)^{-1}
$$

and

$$
\zeta\left(s, \alpha_{j}, \omega_{j} ; \mathfrak{a}_{j l}\right)=\sum_{m=0}^{\infty} \frac{a_{m j l} \omega_{j}(m)}{\left(m+\alpha_{j}\right)^{s}}, \quad j=1, \ldots, r, l=1, \ldots, l_{j} .
$$

Denote by $P_{\underline{\zeta}}$ the distribution of the random element $\underline{\zeta}(\hat{s}, s, \underline{\alpha}, \underline{\omega} ; \underline{\mathfrak{a}}, F)$, i.e., for $A \in \mathcal{B}\left(H^{v}\left(D_{\kappa}, D\right)\right)$,

$$
P_{\underline{\zeta}}(A)=\underline{m}_{H}(\underline{\omega} \in \underline{\Omega}: \underline{\zeta}(\hat{s}, s, \underline{\alpha}, \underline{\omega} ; \underline{\mathfrak{a}}, F) \in A) .
$$

Theorem 4. Suppose that the numbers $\alpha_{1}, \ldots, \alpha_{r}$ are algebraically independent over $\mathbb{Q}$. Then $P_{T}$ converges weakly to $P_{\underline{\zeta}}$ as $T \rightarrow \infty$.

We divide the proof of Theorem 4 into several lemmas. Define

$$
\begin{gathered}
Q_{T}(A)=\nu_{T}\left(\left(\left(p^{-i \tau}: p \in \mathcal{P}\right),\left(\left(m+\alpha_{1}\right)^{-i \tau}: m \in \mathbb{N}_{0}\right), \ldots,\right.\right. \\
\left.\left.\left(\left(m+\alpha_{r}\right)^{-i \tau}: m \in \mathbb{N}_{0}\right)\right) \in A\right), \quad A \in \mathcal{B}(\underline{\Omega}) .
\end{gathered}
$$


Lemma 1. Suppose that the numbers $\alpha_{1}, \ldots, \alpha_{r}$ are algebraically independent over $\mathbb{Q}$. Then $Q_{T}$ converges weakly to the Haar measure $\underline{m}_{H}$ as $T \rightarrow \infty$.

Proof of the lemma is given in [4], Lemma 1.

Let $\sigma_{1}>\frac{1}{2}$ be a fixed number,

$$
\begin{aligned}
& u_{n}(m)=\exp \left\{-(m / n)^{\sigma_{1}}\right\}, \quad m, n \in \mathbb{N}, \\
& u_{n}\left(m, \alpha_{j}\right)=\exp \left\{-\left(\frac{m+\alpha_{j}}{n+\alpha_{j}}\right)^{\sigma_{1}}\right\}, \quad m \in \mathbb{N}_{0}, n \in \mathbb{N} .
\end{aligned}
$$

Define

$$
\varphi_{n}(\hat{s}, F)=\sum_{m=1}^{\infty} \frac{c(m) u_{n}(m)}{m^{\hat{s}}}, \quad \zeta_{n}\left(s, \alpha_{j} ; \mathfrak{a}_{j l}\right)=\sum_{m=0}^{\infty} \frac{a_{m j l} u_{n}\left(m, \alpha_{j}\right)}{\left(m+\alpha_{j}\right)^{s}},
$$

$j=1, \ldots, r, l=1, \ldots, l_{j}$. By a standard method based on the application of the Mellin formula, it is obtained that the series for $\varphi_{n}(\hat{s}, F)$ converges absolutely for $\operatorname{Re} \hat{s}>\frac{\kappa}{2}$, and the series for $\zeta_{n}\left(s, \alpha_{j} ; \mathfrak{a}_{j l}\right)$ converges absolutely for $\sigma>\frac{1}{2}$.

We extend the functions $\hat{\omega}(p)$ to the set $\mathbb{N}$ by the formula

$$
\hat{\omega}(m)=\prod_{p^{l} \| m} \hat{\omega}^{l}(p), \quad m \in \mathbb{N},
$$

where $p^{l} \| m$ means that $p^{l} \mid m$ but $p^{l+1} \nmid m$, and define

$$
\begin{aligned}
& \varphi_{n}(\hat{s}, \hat{\omega}, F)=\sum_{m=1}^{\infty} \frac{c(m) \hat{\omega}(m) u_{n}(m)}{m^{\hat{s}}}, \\
& \zeta_{n}\left(s, \alpha_{j}, \omega_{j} ; \mathfrak{a}_{j l}\right)=\sum_{m=0}^{\infty} \frac{a_{m j l} \omega_{j}(m) u_{n}\left(m, \alpha_{j}\right)}{\left(m+\alpha_{j}\right)^{s}}, \quad j=1, \ldots, r, l=1, \ldots, l_{j} .
\end{aligned}
$$

Clearly, the series for $\varphi_{n}(\hat{s}, \hat{\omega}, F)$ converges absolutely for $\operatorname{Re} \hat{s}>\frac{\kappa}{2}$, and the series for $\zeta_{n}\left(s, \alpha_{j}, \omega_{j} ; \mathfrak{a}_{j l}\right)$ converges absolutely for $\sigma>\frac{1}{2}$. For brevity, we set

$$
\begin{aligned}
\underline{\zeta}_{n}(\hat{s}, s, \underline{\alpha} ; \underline{\mathfrak{a}}, F)= & \left(\varphi_{n}(\hat{s}, F), \zeta_{n}\left(s, \alpha_{1} ; \mathfrak{a}_{11}\right), \ldots, \zeta_{n}\left(s, \alpha_{1} ; \mathfrak{a}_{1 l_{1}}\right),\right. \\
& \left.\ldots, \zeta_{n}\left(s, \alpha_{r} ; \mathfrak{a}_{r 1}\right), \ldots, \zeta_{n}\left(s, \alpha_{r} ; \mathfrak{a}_{r l_{r}}\right)\right)
\end{aligned}
$$

and

$$
\begin{aligned}
\underline{\zeta}_{n}(\hat{s}, s, \underline{\alpha}, \underline{\omega} ; \underline{\mathfrak{a}}, F)= & \left(\varphi_{n}(\hat{s}, \hat{\omega}, F), \zeta_{n}\left(s, \alpha_{1}, \omega_{1} ; \mathfrak{a}_{11}\right), \ldots, \zeta_{n}\left(s, \alpha_{1}, \omega_{1} ; \mathfrak{a}_{1 l_{1}}\right), \ldots,\right. \\
& \left.\zeta_{n}\left(s, \alpha_{r}, \omega_{r} ; \mathfrak{a}_{r 1}\right), \ldots, \zeta_{n}\left(s, \alpha_{r}, \omega_{r} ; \mathfrak{a}_{r l_{r}}\right)\right) .
\end{aligned}
$$

Now, on the space $\left(H^{v}\left(D_{\kappa}, D\right), \mathcal{B}\left(H^{v}\left(D_{\kappa}, D\right)\right)\right)$, define two probability measures

$$
\begin{aligned}
& P_{T, n}(A)=\nu_{T}\left(\underline{\zeta}_{n}(\hat{s}+i \tau, s+i \tau, \underline{\alpha} ; \underline{\mathfrak{a}}, F) \in A\right), \\
& P_{T, n, \underline{\omega}_{0}}(A)=\nu_{T}\left(\underline{\zeta}_{n}\left(\hat{s}+i \tau, s+i \tau, \underline{\alpha}, \underline{\omega}_{0} ; \underline{\mathfrak{a}}, F\right) \in A\right),
\end{aligned}
$$

where $\underline{\omega}_{0}=\left(\hat{\omega}_{0}, \omega_{10}, \ldots, \omega_{r 0}\right)$ is a fixed element of $\underline{\Omega}$. 
Lemma 2. Suppose that the numbers $\alpha_{1}, \ldots, \alpha_{r}$ are algebraically independent over $\mathbb{Q}$. Then the probability measures $P_{T, n}$ and $P_{T, n, \omega_{0}}$ both converge weakly to the same probability measure $P_{n}$ on $\left(H^{v}\left(D_{\kappa}, D\right), \mathcal{B}\left(\bar{H}^{v}\left(D_{\kappa}, D\right)\right)\right)$ as $T \rightarrow \infty$.

Proof. We argue similarly to the proof of Lemma 2 from [4]. The absolute convergence of the series for $\varphi_{n}(\hat{s}, F)$ and $\zeta_{n}\left(s, \alpha_{j} ; \mathfrak{a}_{j l}\right)$ implies the continuity of the function $h_{n}: \underline{\Omega} \rightarrow H^{v}\left(D_{\kappa}, D\right)$ defined by the formula

$$
h_{n}(\underline{\omega})=\underline{\zeta}_{n}(\hat{s}, s, \underline{\alpha}, \underline{\omega} ; \underline{\mathfrak{a}}, F) .
$$

Moreover, we have that

$$
\begin{aligned}
& h_{n}\left(\left(p^{-i \tau}: p \in \mathcal{P}\right),\left(\left(m+\alpha_{1}\right)^{-i \tau}: m \in \mathbb{N}_{0}\right), \ldots,\left(\left(m+\alpha_{r}\right)^{-i \tau}: m \in \mathbb{N}_{0}\right)\right) \\
& \quad=\underline{\zeta}_{n}(\hat{s}+i \tau, s+i \tau, \underline{\alpha} ; \underline{\mathfrak{a}}, F) .
\end{aligned}
$$

Hence, $P_{T, n}=Q_{T} h_{n}^{-1}$. This, the continuity of the function $h_{n}$ and Theorem 5.1 from [1] together with Lemma 1 show that the measure $P_{T, n}$ converges weakly to $P_{n}=\underline{m}_{H} h_{n}^{-1}$ as $T \rightarrow \infty$.

Let the function $g_{n}: \underline{\Omega} \rightarrow H^{v}\left(D_{\kappa}, D\right)$ be given by the formula $g_{n}(\underline{\omega})=$ $h_{n}\left(\underline{\omega} \underline{\omega}_{0}\right)$. Then the above arguments show that the measure $P_{T, n, \underline{\omega}_{0}}$ converges weakly to the measure $\underline{m}_{H} g_{n}^{-1}$ as $T \rightarrow \infty$. However, the invariance of the Haar measure $\underline{m}_{H}$ implies the equality $\underline{m}_{H} h_{n}^{-1}=\underline{m}_{H} g_{n}^{-1}$. This proves the lemma.

For the proof of Theorem 4, we need to pass from $\underline{\zeta}_{n}(\hat{s}, s, \underline{\alpha} ; \underline{\mathfrak{a}})$ to $\underline{\zeta}(\hat{\hat{s}}, s, \underline{\alpha}$; $\underline{\mathfrak{a}})$. This procedure requires the metric on the space $H^{v}\left(D_{\kappa}, D\right)$ which induces its topology of uniform convergence on compacta. It is well known that there exists a sequence $\left\{\hat{K}_{k}: k \in \mathbb{N}\right\}$ of compact subsets of $D_{\kappa}$ and a sequence of compact subsets of $D$ such that $D_{\kappa}=\bigcup_{k=1}^{\infty} \hat{K}_{k}$ and $D=\bigcup_{k=1}^{\infty} K_{k}$. Moreover, the sets $\hat{K}_{k}$ and $K_{k}$ can be chosen to satisfy $\hat{K}_{k} \subset \hat{K}_{k+1}, K_{k} \subset K_{k+1}$ for all $k \in \mathbb{N}$, and, for every compact $\hat{K} \subset D_{\kappa}$ and $K \subset D$, there exist $\hat{k}$ and $k$ such that $\hat{K} \subset \hat{K}_{\hat{k}}$ and $K \subset K_{k}$. For $\hat{f}, \hat{g} \in H\left(D_{\kappa}\right)$, let

$$
\hat{\rho}(\hat{f}, \hat{g})=\sum_{k=1}^{\infty} 2^{-k} \frac{\sup _{s \in \hat{K}_{k}}|\hat{f}(s)-\hat{g}(s)|}{1+\sup _{s \in \hat{K}_{k}}|\hat{f}(s)-\hat{g}(s)|}
$$

and similarly, for $f, g \in H(D)$, let

$$
\rho(f, g)=\sum_{k=1}^{\infty} 2^{-k} \frac{\sup _{s \in K_{k}}|f(s)-g(s)|}{1+\sup _{s \in K_{k}}|f(s)-g(s)|} .
$$

Then $\hat{\rho}$ and $\rho$ are the metrics on $H\left(D_{\kappa}\right)$ and $H(D)$, respectively, which induce the topology of uniform convergence on compacta. For $f=\left(\hat{f}, f_{11}, \ldots, f_{1 l_{1}}, \ldots\right.$, $\left.f_{r 1}, \ldots, f_{r l_{r}}\right), \underline{g}=\left(\hat{g}, g_{11}, \ldots, g_{1 l_{1}}, \ldots, g_{r 1}, \ldots, g_{r l_{r}}\right) \in \bar{H}^{v}\left(D_{\kappa}, D\right)$, define

$$
\rho_{v}(\underline{f}, \underline{g})=\max \left(\hat{\rho}(\hat{f}, \hat{g}), \max _{1 \leq j \leq r} \max _{1 \leq l \leq l_{j}} \rho\left(f_{j l}, g_{j l}\right)\right) \text {. }
$$

Then we have that $\rho_{v}$ is a metric on $H^{v}\left(D_{\kappa}, D\right)$ inducing its topology.

Having the metric on $H^{v}\left(D_{\kappa}, D\right)$, we can approximate in the mean $\underline{\zeta}(\hat{s}, s, \underline{\alpha}$; $\underline{\mathfrak{a}}, F)$ by $\underline{\zeta}_{n}(\hat{s}, s, \underline{\alpha} ; \underline{\mathfrak{a}}, F)$, and $\underline{\zeta}(\hat{s}, s, \underline{\alpha}, \underline{\omega} ; \underline{\mathfrak{a}}, F)$ by $\underline{\zeta}_{n}(\hat{s}, s, \underline{\alpha}, \underline{\omega} ; \underline{\mathfrak{a}}, F)$. 
Lemma 3. The relation

$$
\lim _{n \rightarrow \infty} \limsup _{T \rightarrow \infty} \frac{1}{T} \int_{0}^{T} \rho_{v}\left(\underline{\zeta}(\hat{s}+i \tau, s+i \tau, \underline{\alpha} ; \underline{\mathfrak{a}}, F), \underline{\zeta}_{n}(\hat{s}+i \tau, s+i \tau, \underline{\alpha} ; \underline{\mathfrak{a}}, F)\right) \mathrm{d} \tau=0
$$

holds.

Proof. In [5], it was obtained that, for every compact subset $K \subset D_{\kappa}$,

$$
\lim _{n \rightarrow \infty} \limsup _{T \rightarrow \infty} \frac{1}{T} \int_{0}^{T} \sup _{s \in K}\left|\varphi(s+i \tau, F)-\varphi_{n}(s+i \tau, F)\right| \mathrm{d} \tau=0 .
$$

Hence, we have that

$$
\lim _{n \rightarrow \infty} \limsup _{T \rightarrow \infty} \frac{1}{T} \int_{0}^{T} \hat{\rho}\left(\varphi(\hat{s}+i \tau, F), \varphi_{n}(\hat{s}+i \tau, F)\right) \mathrm{d} \tau=0 .
$$

Similarly, it follows from [6] that

$$
\lim _{n \rightarrow \infty} \limsup _{T \rightarrow \infty} \frac{1}{T} \int_{0}^{T} \max _{1 \leq j \leq r} \max _{1 \leq l \leq l_{j}} \rho\left(\zeta\left(s+i \tau, \alpha_{j} ; \mathfrak{a}_{j l}\right), \zeta_{n}\left(s+i \tau, \alpha_{j} ; \mathfrak{a}_{j l}\right)\right) \mathrm{d} \tau=0 .
$$

This, (1.1) and definition of the metric $\rho_{v}$ prove the lemma.

Lemma 4. Suppose that the numbers $\alpha_{1}, \ldots, \alpha_{r}$ are algebraically independent over $\mathbb{Q}$. Then, for almost all $\underline{\omega} \in \underline{\Omega}$,

$$
\begin{aligned}
\lim _{n \rightarrow \infty} \limsup _{T \rightarrow \infty} \frac{1}{T} \int_{0}^{T} \rho_{v}(\underline{\zeta}(\hat{s}+i \tau, s+i \tau, \underline{\alpha}, \underline{\omega} ; \underline{\mathfrak{a}}, F), \\
\\
\left.\underline{\zeta}_{n}(\hat{s}+i \tau, s+i \tau, \underline{\alpha}, \underline{\omega} ; \underline{\mathfrak{a}}, F)\right) \mathrm{d} \tau=0 .
\end{aligned}
$$

Proof. In [5], it was proved that, for every compact subset $K \subset D_{\kappa}$,

$$
\lim _{n \rightarrow \infty} \limsup _{T \rightarrow \infty} \frac{1}{T} \int_{0}^{T} \sup _{s \in K}\left|\varphi(s+i \tau, \hat{\omega}, F)-\varphi_{n}(s+i \tau, \hat{\omega}, F)\right| \mathrm{d} \tau=0
$$

for almost all $\hat{\omega} \in \hat{\Omega}$. From this, we obtain that, for almost all $\hat{\omega} \in \hat{\Omega}$,

$$
\lim _{n \rightarrow \infty} \limsup _{T \rightarrow \infty} \frac{1}{T} \int_{0}^{T} \hat{\rho}\left(\varphi(\hat{s}+i \tau, \hat{\omega}, F), \varphi_{n}(\hat{s}+i \tau, \hat{\omega}, F)\right) \mathrm{d} \tau=0 .
$$

Similarly, by [6], we have that, for almost all $\left(\omega_{1}, \ldots, \omega_{r}\right) \in \Omega_{1} \times \cdots \times \Omega_{r}$,

$$
\begin{gathered}
\lim _{n \rightarrow \infty} \limsup _{T \rightarrow \infty} \frac{1}{T} \int_{0}^{T} \max _{1 \leq j \leq r} \max _{1 \leq l \leq l_{j}} \rho\left(\zeta\left(s+i \tau, \alpha_{j}, \omega_{j} ; \mathfrak{a}_{j l}\right),\right. \\
\left.\zeta_{n}\left(s+i \tau, \alpha_{j}, \omega_{j} ; \mathfrak{a}_{j l}\right)\right) \mathrm{d} \tau=0 .
\end{gathered}
$$

Since the measure $\underline{m}_{H}$ is the product of the Haar measures on $(\hat{\Omega}, \mathcal{B}(\hat{\Omega}))$, and on $\left(\Omega_{1} \times \cdots \times \Omega_{r}, \overline{\mathcal{B}}\left(\Omega_{1} \times \cdots \times \Omega_{r}\right)\right),(1.2),(1.3)$ and the definition of the metric $\rho_{v}$ imply, for almost all $\underline{\omega} \in \underline{\Omega}$, the equality of the lemma.

For $\underline{\omega} \in \underline{\Omega}$, define one more probability measure

$$
\widetilde{P}_{T}(A) \stackrel{\text { def }}{=} \nu_{T}(\underline{\zeta}(\hat{s}+i \tau, s+i \tau, \underline{\alpha}, \underline{\omega} ; \underline{\mathfrak{a}}, F) \in A), \quad A \in \mathcal{B}\left(H^{v}\left(D_{\kappa}, D\right)\right) .
$$


Lemma 5. Suppose that the numbers $\alpha_{1}, \ldots, \alpha_{r}$ are algebraically independent over $\mathbb{Q}$. Then the probability measures $P_{T}$ and $\widetilde{P}_{T}$ both converge weakly to the same probability measure $P$ on $\left(H^{v}\left(D_{\kappa}, D\right), \mathcal{B}\left(H^{v}\left(D_{\kappa}, D\right)\right)\right)$ as $T \rightarrow \infty$.

Proof. Let $\theta$ be a random variable on a certain probability space $\left(\Omega_{0}, \mathcal{B}\left(\Omega_{0}\right)\right.$, $\mathbb{P})$ which is uniformly distributed on $[0,1]$. On $\left(\Omega_{0}, \mathcal{B}\left(\Omega_{0}\right), \mathbb{P}\right)$, define the $H^{v}\left(D_{\kappa}, D\right)$-valued random element $\underline{X}_{T, n}$ by the formula

$$
\begin{aligned}
& \underline{X}_{T, n}(\hat{s}, s)=\left(X_{T, n}(\hat{s}), X_{T, n, 1,1}(s), \ldots, X_{T, n, 1, l_{1}}(s), \ldots,\right. \\
& \left.X_{T, n, r, 1}(s), \ldots, X_{T, n, r, l_{r}}(s)\right)=\underline{\zeta}_{n}(\hat{s}+i \theta T, s+i \theta T, \underline{\alpha} ; \underline{\mathfrak{a}}, F) .
\end{aligned}
$$

Then Lemma 2 implies the relation

$$
\underline{X}_{T, n}(\hat{s}, s) \underset{T \rightarrow \infty}{\stackrel{\mathcal{D}}{\longrightarrow}} \underline{X}_{n}(\hat{s}, s),
$$

where

$$
\underline{X}_{n}(\hat{s}, s)=\left(X_{n}(\hat{s}), X_{n, 1,1}(s), \ldots, X_{n, 1, l_{1}}(s), \ldots, X_{n, r, 1}(s), \ldots, X_{n, r, l_{r}}(s)\right)
$$

is an $H^{v}\left(D_{\kappa}, D\right)$-valued random element with the distribution $P_{n}$ in the notation of Lemma 2, and, as usual, $\stackrel{\mathcal{D}}{\longrightarrow}$ means the convergence in distribution. We have mentioned above that the series for $\varphi_{n}(s, F)$ converges absolutely for $\sigma>\frac{\kappa}{2}$. Therefore, for $\sigma>\frac{\kappa}{2}$,

$$
\begin{aligned}
\lim _{T \rightarrow \infty} \frac{1}{T} \int_{0}^{T}\left|\varphi_{n}(\sigma+i t, F)\right|^{2} \mathrm{~d} t & =\sum_{m=1}^{\infty} \frac{c^{2}(m) u_{n}^{2}(m)}{m^{2 \sigma}} \\
& \leq \sum_{m=1}^{\infty} \frac{c^{2}(m)}{m^{2 \sigma}}<\infty
\end{aligned}
$$

for all $n \in \mathbb{N}$, because of the Deligne [3] estimate

$$
|c(m)| \ll m^{\frac{\kappa-1}{2}} .
$$

Similarly, the absolute convergence of the series for $\zeta_{n}\left(s, \alpha_{j} ; \mathfrak{a}_{j l}\right)$ shows that, for $\sigma>\frac{1}{2}$,

$$
\begin{aligned}
\lim _{T \rightarrow \infty} \frac{1}{T} \int_{0}^{T}\left|\zeta_{n}\left(\sigma+i t, \alpha_{j} ; \mathfrak{a}_{j l}\right)\right|^{2} \mathrm{~d} t & =\sum_{m=0}^{\infty} \frac{\left|a_{m j l}\right|^{2} u_{n}^{2}\left(m, \alpha_{j}\right)}{\left(m+\alpha_{j}\right)^{2 \sigma}} \\
& \leq \sum_{m=0}^{\infty} \frac{\left|a_{m j l}\right|^{2}}{\left(m+\alpha_{j}\right)^{2 \sigma}}<\infty
\end{aligned}
$$

for all $n \in \mathbb{N}$. Now a simple application of the Cauchy integral formula and (1.5) lead to the inequality

$$
\limsup _{T \rightarrow \infty} \frac{1}{T} \int_{0}^{T} \sup _{s \in \hat{K}_{k}}\left|\varphi_{n}(s+i \tau, F)\right| \mathrm{d} \tau \leq \hat{C}_{k}\left(\sum_{m=1}^{\infty} \frac{c^{2}(m)}{m^{2 \hat{\sigma}_{k}}}\right)^{\frac{1}{2}}, \quad n \in \mathbb{N}
$$


with some $\hat{C}_{k}>0$ and $\hat{\sigma}_{k}>\frac{\kappa}{2}$. Analogically, (1.6) shows that $\limsup _{T \rightarrow \infty} \frac{1}{T} \int_{0}^{T} \sup _{s \in K_{k}}\left|\zeta_{n}\left(s+i \tau, \alpha_{j} ; \mathfrak{a}_{j l}\right)\right| \mathrm{d} \tau \leq C_{k}\left(\sum_{m=0}^{\infty} \frac{\left|a_{m j l}\right|^{2}}{\left(m+\alpha_{j}\right)^{2 \sigma_{k}}}\right)^{\frac{1}{2}}, \quad n \in \mathbb{N}$

with some $C_{k}>0$ and $\sigma_{k}>\frac{1}{2}$. Here $\hat{K}_{k}$ and $K_{k}$ are compact sets from the definition of the metric $\rho_{v}$.

We set

$$
\hat{R}_{k}=\hat{C}_{k}\left(\sum_{m=1}^{\infty} \frac{c^{2}(m)}{m^{2 \hat{\sigma}_{k}}}\right)^{\frac{1}{2}}, \quad R_{j l k}=C_{k}\left(\sum_{m=0}^{\infty} \frac{\left|a_{m j l}\right|^{2}}{\left(m+\alpha_{j}\right)^{2 \sigma_{k}}}\right)^{\frac{1}{2}} .
$$

Then, taking $\hat{M}_{k}=\hat{R}_{k} 2^{k+1} \varepsilon^{-1}$ and $M_{j l k}=R_{j l k} 2^{v_{1}+k+1} \varepsilon^{-1}$, where $k \in \mathbb{N}$ and $\varepsilon>0$ is an arbitrary number, we obtain by (1.7) and (1.8) that

$$
\begin{aligned}
& \limsup _{T \rightarrow \infty} \mathbb{P}\left(\left(\sup _{\hat{s} \in \hat{K}_{k}}\left|X_{T, n}(\hat{s})\right|>\hat{M}_{k}\right) \vee \exists j, l:\left(\sup _{s \in K_{k}}\left|X_{T, n, j, l}(s)\right|>M_{j l k}\right)\right) \\
& \leq \limsup _{T \rightarrow \infty} \mathbb{P}\left(\sup _{\hat{s} \in \hat{K}_{k}}\left|X_{T, n}(\hat{s})\right|>\hat{M}_{k}\right) \\
& \quad+\sum_{j=1}^{r} \sum_{l=1}^{l_{j}} \limsup _{T \rightarrow \infty} \mathbb{P}\left(\sup _{s \in K_{k}}\left|X_{T, n, j, l}(s)\right|>M_{j l k}\right) \\
& \leq \frac{1}{\hat{M}_{k}} \sup _{n \in \mathbb{N}} \limsup _{T \rightarrow \infty} \frac{1}{T} \int_{0}^{T} \sup _{\hat{s} \in \hat{K}_{k}}\left|\varphi_{n}(\hat{s}+i \tau, F)\right| \mathrm{d} \tau \\
& \quad+\sum_{j=1}^{r} \sum_{l=1}^{l_{j}} \frac{1}{M_{j l k}} \sup _{n \in \mathbb{N}} \lim \sup _{T \rightarrow \infty} \frac{1}{T} \int_{0}^{T} \sup _{s \in K_{k}}\left|\zeta_{n}\left(s+i \tau, \alpha_{j} ; \mathfrak{a}_{j l}\right)\right| \mathrm{d} \tau \\
& \leq \frac{\hat{R}_{k}}{\hat{M}_{k}}+\sum_{j=1}^{r} \sum_{l=1}^{l_{j}} \frac{R_{j l k}}{M_{j l k}}=\frac{\varepsilon}{2^{k+1}}+\frac{\varepsilon}{2^{k+1}}=\frac{\varepsilon}{2^{k}} .
\end{aligned}
$$

Using (1.4), hence, we deduce that, for all $n \in \mathbb{N}$,

$$
\mathbb{P}\left(\left(\sup _{\hat{s} \in \hat{K}_{k}}\left|X_{n}(\hat{s})\right|>\hat{M}_{k}\right) \vee \exists j, l:\left(\sup _{s \in K_{k}}\left|X_{n, j, l}(s)\right|>M_{j l k}\right)\right) \leq \frac{\varepsilon}{2^{k}} .
$$

Define a set

$$
\begin{aligned}
H_{\varepsilon}^{v}= & \left\{\left(g, g_{11}, \ldots, g_{1 l_{1}}, \ldots, g_{r 1}, \ldots, g_{r l_{r}}\right) \in H^{v}\left(D_{\kappa}, D\right): \sup _{\hat{s} \in \hat{K}_{k}}|g(\hat{s})| \leq \hat{M}_{k},\right. \\
& \left.\sup _{s \in K_{k}}\left|g_{j l}(s)\right| \leq M_{j l k}, j=1, \ldots, r, l=1, \ldots, l_{j}, k \in \mathbb{N}\right\}
\end{aligned}
$$

Then $H_{\varepsilon}^{v}$ is a compact subset of the space $H^{v}\left(D_{\kappa}, D\right)$. Moreover, in view of $(1.9)$,

$$
\mathbb{P}\left(\underline{X}_{n}(\hat{s}, s) \in H_{\varepsilon}^{v}\right) \geq 1-\varepsilon \sum_{k=1}^{\infty} \frac{1}{2^{k}}=1-\varepsilon
$$


for all $n \in \mathbb{N}$. Thus, by the definition of the random element $\underline{X}_{n}(\hat{s}, s)$,

$$
P_{n}\left(H_{\varepsilon}^{v}\right) \geq 1-\varepsilon
$$

for all $n \in \mathbb{N}$. This means that the family of probability measures $\left\{P_{n}: n \in \mathbb{N}\right\}$ is tight, and, by the Prokhorov theorem, it is relatively compact. Therefore, there exists a subsequence $\left\{P_{n_{k}}\right\} \subset\left\{P_{n}\right\}$ such that $P_{n_{k}}$ converges weakly to a certain probability measure $P$ on $\left(H^{v}\left(D_{\kappa}, D\right), \mathcal{B}\left(H^{v}\left(D_{\kappa}, D\right)\right)\right)$ as $k \rightarrow \infty$. This can be written in the form

$$
\underline{X}_{n_{k}}(\hat{s}, s) \underset{k \rightarrow \infty}{\stackrel{\mathcal{D}}{\longrightarrow}} P
$$

Define one more $H^{v}\left(D_{\kappa}, D\right)$-valued random element $\underline{X}_{T}(\hat{s}, s)$ by the formula

$$
X_{T}(\hat{s}, s)=\underline{\zeta}(\hat{s}+i \theta T, s+i \theta T, \underline{\alpha} ; \underline{\mathfrak{a}}, F) .
$$

Then Lemma 3 shows that, for every $\varepsilon>0$,

$$
\begin{aligned}
\lim _{n \rightarrow \infty} & \limsup _{T \rightarrow \infty} \mathbb{P}\left(\rho_{v}\left(\underline{X}_{T}(\hat{s}, s), \underline{X}_{T, n}(\hat{s}, s)\right) \geq \varepsilon\right) \\
= & \lim _{n \rightarrow \infty} \limsup _{T \rightarrow \infty} \nu_{T}\left(\rho_{v}\left(\underline{\zeta}(\hat{s}+i \tau, s+i \tau, \underline{\alpha} ; \underline{\mathfrak{a}}, F), \underline{\zeta}_{n}(\hat{s}+i \tau, s+i \tau, \underline{\alpha} ; \underline{\mathfrak{a}})\right) \geq \varepsilon\right) \\
\leq & \lim _{n \rightarrow \infty} \limsup _{T \rightarrow \infty} \frac{1}{T \varepsilon} \int_{0}^{T} \rho_{v}(\underline{\zeta}(\hat{s}+i \tau, s+i \tau, \underline{\alpha} ; \underline{\mathfrak{a}}, F), \\
& \left.\underline{\zeta}_{n}(\hat{s}+i \tau, s+i \tau, \underline{\alpha} ; \underline{\mathfrak{a}}, F)\right) \mathrm{d} \tau=0 .
\end{aligned}
$$

This, (1.4), (1.10) and Theorem 4.2 of [1] imply the relation

$$
\underline{X}_{T}(\hat{s}, s) \underset{T \rightarrow \infty}{\stackrel{\mathcal{D}}{\longrightarrow}} P
$$

and thus, $P_{T}$ converges weakly to $P$ as $T \rightarrow \infty$. The relation (1.11) also shows that the measure $P$ is independent of the choice of the sequence $\left\{P_{n_{k}}\right\}$, and this yields the relation

$$
\underline{X}_{n}(\hat{s}, s) \underset{n \rightarrow \infty}{\stackrel{\mathcal{D}}{\longrightarrow}} P .
$$

It remains to show that the measure $\widetilde{P}_{T}$ also converges weakly to $P$ as $T \rightarrow \infty$. We set

$$
\begin{aligned}
& \underline{\widetilde{X}}_{T, n}(\hat{s}, s)=\underline{\zeta}_{n}(\hat{s}+i \theta T, s+i \theta T, \underline{\alpha}, \underline{\omega} ; \underline{\mathfrak{a}}, F), \\
& \underline{\tilde{X}}_{T}(\hat{s}, s)=\underline{\zeta}(\hat{s}+i \theta T, s+i \theta T, \underline{\alpha}, \underline{\omega} ; \underline{\mathfrak{a}}, F) .
\end{aligned}
$$

Then the above arguments together with Lemmas 2 and 4, and relation (1.12) applied for the random elements $\underline{\widetilde{X}}_{T, n}(\hat{s}, s)$ and $\underline{\widetilde{X}}_{T}(\hat{s}, s)$ show that the measure $\widetilde{P}_{T}$ also converges weakly to $P$ as $T \rightarrow \infty$.

In order to prove Theorem 4, it suffices to show that the limit measure $P$ in Lemma 5 is the distribution of the random element $\underline{\zeta}(\hat{s}, s, \underline{\alpha}, \underline{\omega} ; \underline{\mathfrak{a}}, F)$. Define $\underline{\Phi}_{\tau}(\underline{\omega})=\underline{a}_{\tau} \underline{\omega}, \underline{\omega} \in \underline{\Omega}$, where $\underline{a}_{\tau}=\left\{\left(p^{-i \tau}: p \in \mathcal{P}\right),\left(\left(m+\alpha_{1}\right)^{-i \tau}: m \in \mathbb{N}_{0}\right), \ldots\right.$, 
$\left.\left(\left(m+\alpha_{r}\right)^{-i \tau}: m \in \mathbb{N}_{0}\right)\right\}$ for $\tau \in \mathbb{R}$. Then $\left\{\underline{\Phi}_{\tau}: \tau \in \mathbb{R}\right\}$ is a one-parameter group of measurable measure preserving transformations on $\underline{\Omega}$. A set $A \in \mathcal{B}(\underline{\Omega})$ is called invariant with respect to this group if, for every $\tau \in \mathbb{R}$, the sets $A$ and $\underline{\Phi}_{\tau}(A)$ may differ one from another only by $\underline{m}_{H}$-measure zero. The group $\left\{\underline{\Phi}_{\tau}: \tau \in \mathbb{R}\right\}$ is ergodic if its $\sigma$-field of invariant sets consists only of the sets having $\underline{m}_{H}$-measure zero or one.

Lemma 6. Suppose that the numbers $\alpha_{1}, \ldots, \alpha_{r}$ are algebraically independent over $\mathbb{Q}$. Then the group $\left\{\underline{\Phi}_{\tau}: \tau \in \mathbb{R}\right\}$ is ergodic.

Proof of the lemma is given in [7, Lemma 7].

Proof of Theorem 4. We fix a continuity set $A$ of the limit measure $P$ in Lemma 5. Then, using an equivalent of the weak convergence of probability measures in terms of continuity sets, Theorem 2.1 of [1], we have by Lemma 5 that

$$
\lim _{T \rightarrow \infty} \nu_{T}(\underline{\zeta}(\hat{s}+i \tau, s+i \tau, \underline{\alpha}, \underline{\omega} ; \underline{\mathfrak{a}}, F) \in A)=P(A) .
$$

On the probability space $\left(\underline{\Omega}, \mathcal{B}(\underline{\Omega}), \underline{m}_{H}\right)$, define the random variable $\xi(\underline{\omega})$ by the formula

$$
\xi(\underline{\omega})= \begin{cases}1 & \text { if } \underline{\zeta}(\hat{s}, s, \underline{\alpha}, \underline{\omega} ; \underline{\mathfrak{a}}, F) \in A, \\ 0 & \text { otherwise. }\end{cases}
$$

Then the expectation

$$
\mathbb{E} \xi=\underline{m}_{H}(\underline{\omega} \in \underline{\Omega}: \underline{\zeta}(\hat{s}, s, \underline{\alpha}, \underline{\omega} ; \underline{\mathfrak{a}}, F) \in A)=P_{\underline{\zeta}}(A),
$$

where $P_{\underline{\zeta}}$ is the distribution of the random element $\underline{\zeta}(\hat{s}, s, \underline{\alpha}, \underline{\omega} ; \underline{\mathfrak{a}}, F)$. Lemma 6 implies the ergodicity of the process $\xi\left(\underline{\Phi}_{\tau}(\underline{\omega})\right)$. Therefore, by the BirkhoffKhintchine theorem, see, for example, [2], for almost all $\underline{\omega} \in \underline{\Omega}$,

$$
\lim _{T \rightarrow \infty} \frac{1}{T} \int_{0}^{T} \xi\left(\underline{\Phi}_{\tau}(\underline{\omega})\right) \mathrm{d} \tau=\mathbb{E} \xi
$$

However, by the the definitions of $\xi$ and $\underline{\Phi}_{\tau}$, we have that

$$
\frac{1}{T} \int_{0}^{T} \xi\left(\underline{\Phi}_{\tau}(\underline{\omega})\right) \mathrm{d} \tau=\nu_{T}(\underline{\zeta}(\hat{s}+i \tau, s+i \tau, \underline{\alpha}, \underline{\omega} ; \underline{\mathfrak{a}}, F) \in A) .
$$

Therefore, taking into account (1.14) and (1.15), we obtain that

$$
\lim _{T \rightarrow \infty} \nu_{T}(\underline{\zeta}(\hat{s}+i \tau, s+i \tau, \underline{\alpha}, \underline{\omega} ; \underline{\mathfrak{a}}, F) \in A)=P_{\underline{\zeta}}(A) .
$$

This and (1.13) show that $P(A)=P_{\underline{\zeta}}(A)$. Since $A$ is an arbitrary continuity set $A$ of $P$, hence, $P(A)=P_{\zeta}(A)$ for all continuity sets of $P$. Therefore, $P(A)=P_{\underline{\zeta}}(A)$ for all $A \in \mathcal{B}\left(\bar{H}^{v}\left(D_{\kappa}, D\right)\right)$ because all continuity sets form a determining class, see [1]. This completes the proof of Theorem 4. 


\section{The Support of the Measure $P_{\underline{\zeta}}$}

For the proof of the Theorem 3, we need the support of the measure $P_{\zeta}$. Since the space $H^{v}\left(D_{\kappa}, D\right)$ is separable, the support of $P_{\zeta}$ is a minimal closed set $S_{P_{\underline{\zeta}}}$ of $H^{v}\left(D_{\kappa}, D\right)$ such that $P_{\underline{\zeta}}\left(S_{P_{\zeta}}\right)=1$. The set $S_{P_{\zeta}}$ consists of all points $\underline{g} \in H^{v}\left(D_{\kappa}, D\right)$ such that $P_{\underline{\zeta}}(G)>0$ for every open neighbourhood $G$ of $\underline{g}$.

Let

$$
S_{\kappa}=\left\{g \in H\left(D_{\kappa}\right): g(s) \neq 0 \text { or } g(s) \equiv 0\right\} .
$$

Theorem 5. Suppose that $\alpha_{1}, \ldots, \alpha_{r}$ are algebraically independent over $\mathbb{Q}$, and that $\operatorname{rank}\left(B_{j}\right)=l_{j}, j=1 \ldots, r$. Then the support of $P_{\underline{\zeta}}$ is the set $S_{\kappa} \times H^{v_{1}}(D)$.

Proof. By the definition,

$$
H^{v}\left(D_{\kappa}, D\right)=H\left(D_{\kappa}\right) \times H^{v_{1}}(D) .
$$

Since the spaces $H\left(D_{\kappa}\right)$ and $H^{v_{1}}(D)$ are separable, it suffices [1] to consider $P_{\underline{\zeta}}(A)$ for $A=B \times C$, where $B \in \mathcal{B}\left(H\left(D_{\kappa}\right)\right)$ and $C \in \mathcal{B}\left(H^{v_{1}}(D)\right)$. The Haar measure $\underline{m}_{H}$ is the product of the Haar measures $\hat{m}_{H}$ and $m_{H}^{r}$ on $(\hat{\Omega}, \mathcal{B}(\hat{\Omega}))$ and $\left(\Omega_{1} \times \cdots \times \Omega_{r}, \mathcal{B}\left(\Omega_{1} \times \cdots \times \Omega_{r}\right)\right)$, respectively. Therefore, we have that, for $A=B \times C \in \mathcal{B}\left(H^{v}\left(D_{\kappa}, D\right)\right)$,

$$
\begin{aligned}
P_{\underline{\zeta}}(A)= & \underline{m}_{H}(\underline{\omega} \in \underline{\Omega}: \underline{\zeta}(\hat{s}, s, \underline{\alpha}, \underline{\omega} ; \underline{\mathfrak{a}}, F) \in A) \\
= & \underline{m}_{H}\left(\underline{\omega} \in \underline{\Omega}: \varphi(\hat{s}, \hat{\omega}, F) \in B,\left(\zeta\left(s, \alpha_{1}, \omega_{1} ; \mathfrak{a}_{11}\right), \ldots,\right.\right. \\
& \left.\left.\zeta\left(s, \alpha_{1}, \omega_{1} ; \mathfrak{a}_{1 l_{1}}\right), \ldots, \zeta\left(s, \alpha_{r}, \omega_{r} ; \mathfrak{a}_{r 1}\right), \ldots, \zeta\left(s, \alpha_{r}, \omega_{r} ; \mathfrak{a}_{r l_{r}}\right)\right) \in C\right) \\
= & \hat{m}_{H}(\hat{\omega} \in \hat{\Omega}: \varphi(\hat{s}, \hat{\omega}, F) \in B) \\
& \times m_{H}^{r}\left(\left(\omega_{1}, \ldots, \omega_{r}\right) \in \Omega_{1} \times \cdots \times \Omega_{r}:\left(\zeta\left(s, \alpha_{1}, \omega_{1} ; \mathfrak{a}_{11}\right), \ldots,\right.\right. \\
& \left.\left.\zeta\left(s, \alpha_{1}, \omega_{1} ; \mathfrak{a}_{1 l_{1}}\right), \ldots, \zeta\left(s, \alpha_{r}, \omega_{r} ; \mathfrak{a}_{r 1}\right), \ldots, \zeta\left(s, \alpha_{r}, \omega_{r} ; \mathfrak{a}_{r l_{r}}\right)\right) \in C\right) .
\end{aligned}
$$

In [5], it was obtained that the support of the random element $\varphi(\hat{s}, \hat{\omega}, F)$ is the set $S_{\kappa}$, i.e., $S_{\kappa}$ is a minimal closed subset of $H\left(D_{\kappa}\right)$ such that

$$
\hat{m}_{H}\left(\hat{\omega} \in \hat{\Omega}: \varphi(\hat{s}, \hat{\omega}, F) \in S_{\kappa}\right)=1 .
$$

Also, in [6], it was proved that $H^{v_{1}}(D)$ is the support of the random element

$$
\begin{aligned}
& \left(\zeta\left(s, \alpha_{1}, \omega_{1} ; \mathfrak{a}_{11}\right), \ldots, \zeta\left(s, \alpha_{1}, \omega_{1} ; \mathfrak{a}_{1 l_{1}}\right), \ldots, \zeta\left(s, \alpha_{r}, \omega_{r} ; \mathfrak{a}_{r 1}\right), \ldots,\right. \\
& \left.\quad \zeta\left(s, \alpha_{r}, \omega_{r} ; \mathfrak{a}_{r l_{r}}\right)\right)
\end{aligned}
$$

i.e., $H^{v_{1}}(D)$ is a minimal closed set of $H^{v_{1}}(D)$ such that

$$
\begin{aligned}
& m_{H}^{r}\left(\left(\omega_{1}, \ldots, \omega_{r}\right) \in \Omega_{1} \times \cdots \times \Omega_{r}:\left(\zeta\left(s, \alpha_{1}, \omega_{1} ; \mathfrak{a}_{11}\right), \ldots, \zeta\left(s, \alpha_{1}, \omega_{1} ; \mathfrak{a}_{1 l_{1}}\right),\right.\right. \\
& \left.\left.\ldots, \zeta\left(s, \alpha_{r}, \omega_{r} ; \mathfrak{a}_{r 1}\right), \ldots, \zeta\left(s, \alpha_{r}, \omega_{r} ; \mathfrak{a}_{r l_{r}}\right)\right) \in H^{v_{1}}(D)\right)=1 .
\end{aligned}
$$

Therefore, the theorem is a result of $(2.1)-(2.3)$. 


\section{Proof of Theorem 3}

We start with the Mergelyan theorem on the approximation of analytic functions by polynomials.

Lemma 7. Let $K$ be a compact subset of the complex plane with connected complement, and let $f(s)$ be a continuous function on $K$ which is analytic in the interior of $K$. Then, for every $\varepsilon>0$, there exists a polynomial $p(s)$ such that

$$
\sup _{s \in K}|f(s)-p(s)|<\varepsilon .
$$

Proof is given in [8], [9].

Proof of Theorem 3. By Lemma 7, there exist polynomials $p(s)$ and $p_{j l}(s)$ such that

$$
\sup _{s \in K}|f(s)-p(s)|<\frac{\varepsilon}{4}
$$

and

$$
\sup _{1 \leq j \leq r} \sup _{1 \leq l \leq l_{j}} \sup _{s \in K_{j l}}\left|f_{j l}(s)-p_{j l}(s)\right|<\frac{\varepsilon}{2} .
$$

Since $f(s) \neq 0$ on $K, p(s) \neq 0$ on $K$ as well if $\varepsilon$ is small enough. Thus, on $K$ we can define a continuous branch of $\log p(s)$ which will be analytic in the interior of $K$. Therefore, by Lemma 7 , there exists a polynomial $q(s)$ such that

$$
\sup _{s \in K}\left|p(s)-\mathrm{e}^{q(s)}\right|<\frac{\varepsilon}{4} .
$$

This together with (3.1) shows that

$$
\sup _{s \in K}\left|f(s)-\mathrm{e}^{q(s)}\right|<\frac{\varepsilon}{2} .
$$

Define

$$
\begin{aligned}
G= & \left\{\left(g, g_{11}, \ldots, g_{1 l_{1}}, \ldots, g_{r 1}, \ldots, g_{r l_{r}}\right) \in H^{v}\left(D_{\kappa}, D\right):\right. \\
& \left.\sup _{s \in K}\left|g(s)-\mathrm{e}^{q(s)}\right|<\frac{\varepsilon}{2}, \sup _{1 \leq j \leq r} \sup _{1 \leq l \leq l_{j}} \sup _{s \in K_{j l}}\left|g_{j l}(s)-p_{j l}(s)\right|<\frac{\varepsilon}{2}\right\} .
\end{aligned}
$$

In view of Theorem $5,\left(\mathrm{e}^{q(s)}, p_{11}(s), \ldots, p_{1 l_{1}}(s), \ldots, p_{r 1}(s), \ldots, p_{r l_{r}}(s)\right)$ is an element of the support of the measure $P_{\zeta}$. Since the set $G$ is open, hence, we have that $P_{\underline{\zeta}}(G)>0$. Therefore, by Theorem 4 and an equivalent of the weak convergence of probability measures in terms of open sets (Theorem 2.1 of [1]), we obtain that

$$
\begin{aligned}
& \liminf _{T \rightarrow \infty} \nu_{T}\left(\sup _{s \in K}\left|\varphi(s+i \tau, F)-\mathrm{e}^{q(s)}\right|<\frac{\varepsilon}{2},\right. \\
& \left.\quad \sup _{1 \leq j \leq r} \sup _{1 \leq l \leq l_{j}} \sup _{s \in K_{j l}}\left|\zeta\left(s+i \tau, \alpha_{j} ; \mathfrak{a}_{j l}\right)-p_{j l}(s)\right|<\frac{\varepsilon}{2}\right)>0 .
\end{aligned}
$$

Combining this with (3.2) and (3.3) completes the proof of the theorem. 


\section{References}

[1] P. Billingsley. Convergence of Probability Measures. John Wiley \& Sons, New York, 1968.

[2] H. Cramér and M.R. Leadbetter. Stationary and Related Stochastic Processes. Willey, New York, 1967.

[3] P. Deligne. La conjecture de Weil. Inst. Hautes Études Sci. Publ. Math., 43:273307, 1974.

[4] J. Genys, R. Macaitienè, S. Račkauskienè and D. Šiaučiūnas. A mixed joint universality theorem for zeta-functions. Math. Model. Anal., 15(4):431-446, 2010. http://dx.doi.org/10.3846/1392-6292.2010.15.431-446.

[5] A. Laurinčikas and K. Matsumoto. The universality of zeta-functions attached to certain cusp forms. Acta Arith., 98:345-359, 2001.

http://dx.doi.org/10.4064/aa98-4-2.

[6] A. Laurinčikas and S. Skerstonaitè. Joint universality for periodic Hurwitz zetafunctions. II. In R. Steuding and J. Steuding(Eds.), New Dirrections in Value Distribution Theory of Zeta and L-Functions, pp. 161-170, Aachen, 2009. Shaker Verlag.

[7] A. Laurinčikas. Joint universality of zeta-functions with periodic coefficients. $I z v$. RAN, Ser. Matem., 74(3):79-102, 2010. http://dx.doi.org/10.4213/im2771. (In Russian)

[8] S.N. Mergelyan. Uniform approximations to functions of complex variable. Usp. Mat. Nauk, 7:31-122, 1952. (In Russian)

[9] J.L. Walsh. Interpolation and Approximation by Rational Functions in the Complex Domain, vol. 20. Amer. Math. Soc. Coll. Publ., 1960. 